\title{
Forecasting COVID-19 Cases With Government Intervention Comparisons: Lockdown, Trackdown, and Herd Immunity
}

\author{
Esther Lee \\ California Baptist University \\ Jinook Jeong \\ Yonsei University
}

Coronavirus Disease 2019 (COVID19) has been a global public health threat. For countries battling the coronavirus pandemic, stringent social or physical distancing (referred to as lockdown), contact tracking in real-time (referred to as trackdown), and/or herd immunity measures have been used to flatten the COVID-19 epidemic curve. This study aimed to examine the effect of lockdown, trackdown, and herd immunity on daily COVID19 cases and related deaths using time series data from Jan 22, 2020 to Nov 05, 2020. The Susceptible-Infectious-Recovered (SIR) model was applied to characterize the epidemic dynamics and to predict possible contagion scenarios of COVID-19. Optimized parameter values were used to estimate the basic reproductive number (R0) for Sweden, South Korea, Germany, and the United States. Our results suggested that lockdown and trackdown measures have successfully controlled the outbreak and transmission of COVID-19, and herd immunity was able to prevent the spread of COVID-19 in Sweden. However, even if the proportion of immunized individuals in the population as a whole may surpass the herd immunity threshold, these pockets of susceptible individuals are still at risk for local outbreaks as immunity is unevenly distributed within a population. This study also found that lockdowns should remain in place for at least 90 days to prevent the epidemic growth, and both social distancing and public health measures could potentially remove the need for the lockdowns. The fewer confirmed cases and deaths were ascertained by trackdowns.

Keywords: COVID-19, SIR modeling, her immunity, trackdown, lockdown

\section{INTRODUCTION}

Since the COVID-19 outbreak was first detected in Wuhan, China, it has become a pandemic declared by World Health Organization (WHO) on March 11, 2020, which spread to over 200 countries (Wu et al., 2020; Wang et al., 2020; Chan et al., 2020; WHO, 2020). The COVID-19 pandemic has represented many of the characteristics previously identified in infectious disease 'nightmare scenarios,' including the 'silent man' phenomenon. Its impact on the global economy is beyond anything experienced in nearly a century. Many experts warn that the virus will continue to pose a public health threat repeatedly and brace for the coronavirus's multiple waves (Barro et al., 2020; WHO, 2020; McKibbin et al., 2020; Hui et al., 2020).

For battling the coronavirus pandemic, governments have implemented a wide range of nonpharmaceutical interventions (NPIs) to control the spread of COVID-19, reduce the stress on the health 
system, and gain time to develop and produce vaccines under rapidly changing and unpredicted circumstances (Desvars-Larrive et al. 2020; Anderson et al., 2020; Ugarov 2020; Pan et al., 2020; Rocklov, 2020; Sjödin et al., 2020). Government responses to COVID-19 included the herd-immunity strategy, which implies doing nothing to minimum interventions relying on voluntary compliance, and more aggressive approaches such as lockdown and trackdown, sometimes limiting civil rights and liberty.

Government control policies have shown deviations in the timeline of implementation and the prioritization of NPIs. For example, Sweden's initial response is the herd-immunity approach, and thus most businesses stayed open while the world's biggest economies shut down. However, Sweden has revealed that despite adopting the herd immunity approach, only $7.3 \%$ of the Stockholm populations had developed the antibodies by late April 2020 (Public Health Agency of Sweden, 2020), which indicates that Sweden was nowhere near herd immunity. Stringent social and physical distancing, cordon sanitaire, and quarantine (also referred to as lockdown) and even contact tracking in real-time (also referred to as trackdown) measures have flattened the COVID-19 epidemic curve in several other Asian countries (Korea, China, Taiwan, and Singapore) in the early stage of the pandemic. Following the frontier Asian success, governments worldwide have been forced to pursue aggressive policies to stem the spread of this deadly disease and bend the death curve (Desvars-Larrive et al., 2020; Cheng, 2020; Hale et al., 2020; Zheng, 2020).

This study was carried out during the crucial development phase of the pandemic (from Jan 22, 2020 to Nov 05,2020 ) to assess the effectiveness of lockdown, trackdown, and herd immunity to control daily COVID19 cases and related deaths. Specifically, the COVID-19 trajectories in various countries, represented by Sweden (herd immunity), USA (lockdown), Germany (lockdown), and South Korea (trackdown), were explored based on the Susceptible-Infectious-Recovered (SIR) model (Wang et al., 2020; Wangping et al., 2020; Kucharski et al., 2020; Fanelli et al., 2020).

The SIR model deals with time series of dynamic ordinary differential equations in which the total population is divided into three components of being susceptible to the disease (Susceptible), actively infected with the disease (Infectious), and recovered (or dead) and no longer contagious (Recovered). According to the model, whether or not the number of infected individuals is increasing or decreasing at any time $t$ depends on the reproductive ratio ( $R 0$ or R-nought) that signifies the average number of infectious who pass the virus to others. Consequently, by comparing R0s from various countries, we have assessed the effectiveness of government interventions.

Another objective of this study is to help policymakers generate the social and economic scenarios related to the dynamics of the spread of COVID-19 by obtaining estimates for the number of susceptible, infectious, and recovered. While the stringent measures based on lockdown and/or trackdown have been highly successful in controlling catastrophic epidemic escalation, they impose inevitable social and economic costs on society.

Based on recognizing that no country was yet 'safe' from the spread of the outbreak of COVID-19, this study allows for quantitative statements regarding the timing of often haphazard government interventions to support smooth market re-functioning.

The paper is organized as follows. In Section 2, we introduce the SIR model in the context of the ordinary differential equations system, discuss its various aspects, and derive the R0 number as the system's equilibria. In Section 3, we explain the approaches used to study the data and provide the descriptive analysis of the data. We present the results of our analysis in Section 4 and discuss the effectiveness of

government interventions implemented in Sweden, USA, Germany, and South Korea. Finally, in Section 5 , we conclude our work and discuss the policy implications based on comparisons between government interventions.

\section{METHODS}

\section{Overview of the SIR Model}

We employed the SIR model widely used in the analysis of infectious diseases such as Ebola and SARS (Zhang, 2007; Venkatasen et al., 2020; Rachah and Torres, 2015). The SIR model simulates the trajectories 
of an epidemic phenomenon and provides the prediction of the number of COVID-19 cases. In the classic form, it models the mutual and dynamic interaction of people between three different conditions:

- Susceptible $\mathrm{S}(\mathrm{t})$, which denotes individuals who are susceptible to catching the virus, might become infectious if exposed.

- Infectious $I(t)$, which denotes infectious individuals who are suffering from the symptoms of COVID-19 and can spread the virus through contact with the susceptible class of individuals

- Recovered or dead $\mathrm{R}(\mathrm{t})$, which denotes individuals who have immunity to the infection and, consequently, do not affect the transmission dynamics when in contact with other individuals.

This model assumes no new transmissions from animals, no differences in individual immunity, and natural birth and death. The recovered or dead (R) are generally not re-introduced into the susceptible (S) category if it is supposed that they became immune to the disease. This aspect is intensely debated, as in some countries, the second infection of recovered people has been recorded. Despite the strong assumptions on the SIR model, it is known that this model works appropriately in the analysis of the COVID-19 pandemic.

A characteristic of the model is that the sum of the three categories is equal to the total population $(\mathrm{N})$ at any time:

$N=S(t)+I(t)+R(t)$

Hence, the classical SIR model can be described by a series of ordinary differential equations:

$\frac{\mathrm{dS}(\mathrm{t})}{\mathrm{dt}}=-\beta \mathrm{I}(\mathrm{t}) \mathrm{S}(\mathrm{t})$
$\frac{d I(t)}{d t}=\beta I(t) S(t)-\gamma I(t)$
$\frac{d R(t)}{d t}=\gamma I(t)$

It has been observed that in many communities, a spike in the number of infected individuals, I, may occur, which results in a surge in the susceptible population, $\mathrm{S}$. The evolution of the infected population I is governed by the second ODE in the system in (2), where $\beta(>0)$ is the transmission rate and $\gamma(>0)$ is the removal rate calculated by the inverse of the infectious period.

\section{Analysis of the Equilibria}

Let us find the equilibrium points of the system of equations (2) that describes the model. By setting the right-hand side of (2) to zero, we can get:

$$
\frac{\beta}{y} S=1
$$

and define the basic (or effective) reproductive rate:

$$
R_{0}=\frac{\beta}{\gamma}
$$

It is known that the disease will start to decrease monotonically to zero if $\mathrm{R} \_0 \mathrm{~S}<1$, and if greater than one, it will increase (Anderson and May; 1982). Thus, the effective reproductive (referred to as R_0) rate is considered a threshold determining whether an infectious disease will die out quickly or lead to an epidemic.

The optimization solver minimizes an objective function, which is set to decrease the misfit between observed data and calculated responses of I and $\mathrm{R}$ by varying the parameters. Both $\beta$ and $\gamma$ are randomly 
initialized at the local minima, and all individuals are considered susceptible. R software (version 3.6.3) was applied for all the calculations and estimates in the study.

\section{DATA ANALYSIS}

\section{COVID-19 Database}

The analysis is based on the data collected and made by the Center for Systems Science and Engineering at John Hopkins University Center (JHU CSSE, resource: https://coronavirus.jhu.edu/map.html). They represent an official database collected from various official organizations such as the World Health Organization (WHO), the European Centre for Disease Prevention and Control (ECDC), the USA Centers for Disease Control and Prevention, and other organizations.

The data for Sweden, the USA, Germany, and South Korea are organized in the form of a time series where the rows are recorded as in time (from January to November 2020), and the columns include the daily confirmed cases, deaths, and recovered. Table 1 shows the cumulative confirmed cases, deaths, population, the proportion of the confirmed population, the proportion of deaths, and GDP per capita of the four countries.

\section{TABLE 1}

\section{CUMULATIVE CONFIRMED CASES (BY NOVEMBER 5TH, 2020)}

\begin{tabular}{|c|r|r|r|r|r|r|}
\hline Country & $\begin{array}{c}\text { Confirmed } \\
\text { Cases }\end{array}$ & Deaths & Population & $\begin{array}{l}\text { Confirmed/ } \\
\text { Population }\end{array}$ & $\begin{array}{c}\text { Deaths/ } \\
\text { Population }\end{array}$ & GDP/capita (\$) \\
\hline Sweden & 973,604 & 14,048 & $10,278,887$ & $9 \%$ & $0.001 \%$ & $58,012.96$ \\
\hline South Korea & 123,728 & 1,840 & $51,709,098$ & $\leq 1 \%$ & $0.00 \%$ & $28,675.03$ \\
\hline Germany & $3,432,676$ & 83,605 & $83,092,962$ & $4 \%$ & $0.001 \%$ & $47,446.73$ \\
\hline $\begin{array}{c}\text { United } \\
\text { States }\end{array}$ & $32,421,641$ & 577,566 & $328,239,523$ & $10 \%$ & $0.002 \%$ & $55,753.14$ \\
\hline
\end{tabular}

Resource: JHU CSSE; WHO

Considering the vast population gap between the United States and Sweden, the similar confirmed proportions of the two countries imply that Sweden took the risk of allowing a critical mass of infection to open most businesses. Even though both South Korea and Germany have a noticeable population difference from Sweden, they have smaller confirmed proportions. As a result, it is critical to revisit the effectiveness of the different government interventions in the four countries.

\section{Government Control Strategies Database}

Desvars-Larrive et al. (2020) provide the Complexity Science Hun COVID-19 Control Strategies List (CCCSL) dataset that includes non-pharmaceutical interventions taken by the government for 57 countries. They partitioned the countries into the eight tiers based on the number of measures implemented at the different pandemic states by using the K-mean cluster algorithm. Figure 1 shows the eight different tiers of country clusters based on the control strategy timelines. Specifically, anticipatory measures denote those implemented before the day when 10 cases were reported; early measures as those implemented between the day of having 10 cases and the day of having 200 cases; and late measures as those implemented after the day when 200 cases were reported. 


\section{FIGURE 1 \\ COUNTRY-CLUSTERING BASES ON K-MEAN ALGORITHM}

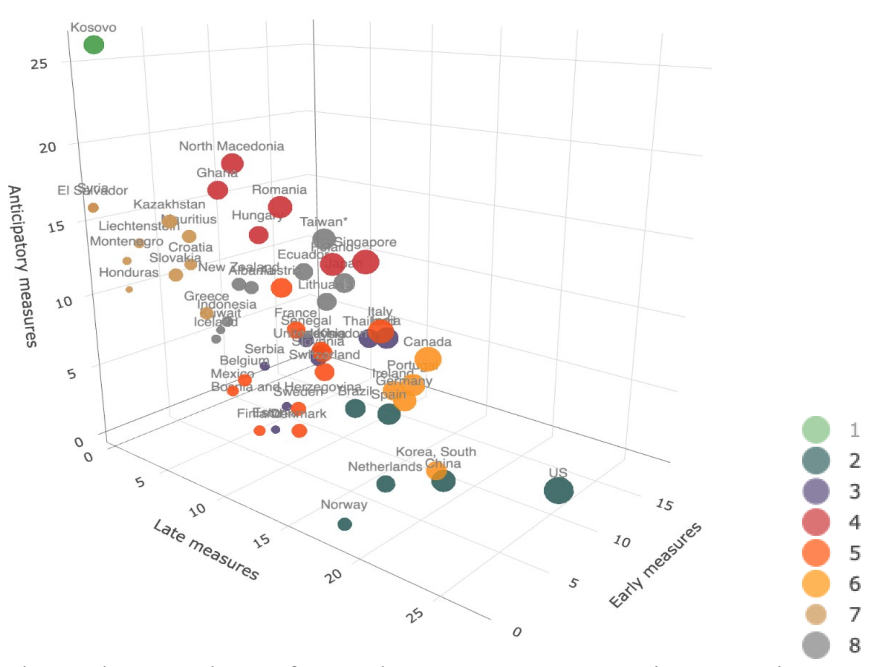

Country-cluster analysis is based on the number of mandatory government interventions and respective dates of implementation, as calculated using the epidemic age ( $\mathrm{t} 0=$ day when 10 cases were reported). Code resource: http://covid19-interventions.com/CountryClusters.html

In order to clarify the calendar time of the government interventions, we also used the government measures dataset provided by the Assessment Capacities Project (ACAPS; Resource: https://www.acaps.org/covid-19-government-measures-dataset). ACAPS, a humanitarian analysis nonprofit, classified all the non-pharmaceutical measures (NPIs) implemented by governments worldwide into five categories:

- Public health measures: obligations to wear masks in the public space, hand sanitization campaigns, etc.

- Movement restrictions: international travel restrictions, domestic travel restrictions, curfew, etc.

- Governance and social and economic measures: bans on mass gatherings, bans on sporting and recreational events, restaurant and bar closures, declarations of states of emergency, public testing, enhanced surveillance, school closures, etc.

- Lockdowns: domestic lockdowns

- Social distancing

where each category is broken down into several types of measures.

It is worthwhile to note that the tidycovid19 package facilitates the download of the related data from various sources including the JHU CSSE and ACAPS as:

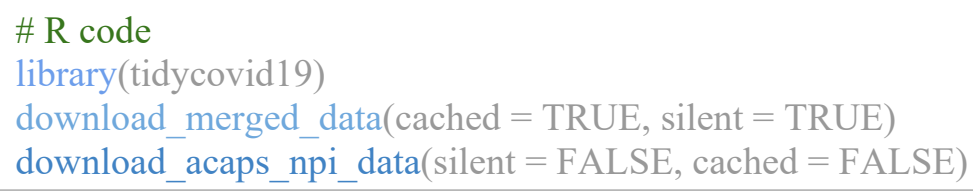

Combining the country-cluster classification and the ACAPS datasets, we specified the four countries into Tier 2, 5, and 6 and rearranged them into herd immunity (i.e., small interventions), trackdown, and lockdown (i.e., strong intervention) depending on the intervention types. Table 2 shows that Sweden (Tier 5) sets a seductive example of minimizing the stringent government interventions, whereas the rigorous social or physical government interventions have been found in Germany (Tier 6) and the United States 
(Tier 2). Compared to the frequencies of government interventions taken by Germany that is in the same Tier, South Korea took relatively fewer interventions. However, South Korea uniquely implemented measures to identify cases, track the contacts in real-time that have been obtaining great caution due to its danger to put personal privacy at risk, and severely isolate suspected cases.

TABLE 2

COUNTRY-CLUSTERING BASED ON THE NUMBER OF NPIS

\begin{tabular}{|c|c|c|c|c|c|c|}
\hline $\begin{array}{c}\text { Intervention } \\
\text { type }\end{array}$ & $\begin{array}{c}\text { Country } \\
\text { Cluster }\end{array}$ & Country & N(A) & N(E) & N(L) & Total \\
\hline small & Tier 5 & Sweden & 5 & 3 & 13 & 21 \\
\hline trackdown & Tier 6 & South Korea & 0 & 9 & 18 & 27 \\
\hline strong & Tier 6 & Germany & 6 & 6 & 18 & 30 \\
\hline strong & Tier 2 & United States & 3 & 7 & 27 & 37 \\
\hline
\end{tabular}

Note: Anticipatory measures (A), Early measures (E), and Late measures (L).

\section{RESULTS}

\section{Government Interventions (NPIs) Distributions Over Time}

Here we present how the government interventions distribute across calendar time. Figure 2 shows that the government interventions were intensely implemented in the first 200 days (late February thru early August), right before the pandemic's first wave peaked. The number of interventions after late August remained slightly low compared to those in the first 200 days, but an occasionally high intervention was detected during the holiday season.

\section{FIGURE 2 \\ HISTOGRAM OF NPIS ACROSS CALENDAR TIME (END TIME: NOVEMBER 5th, 2020)}

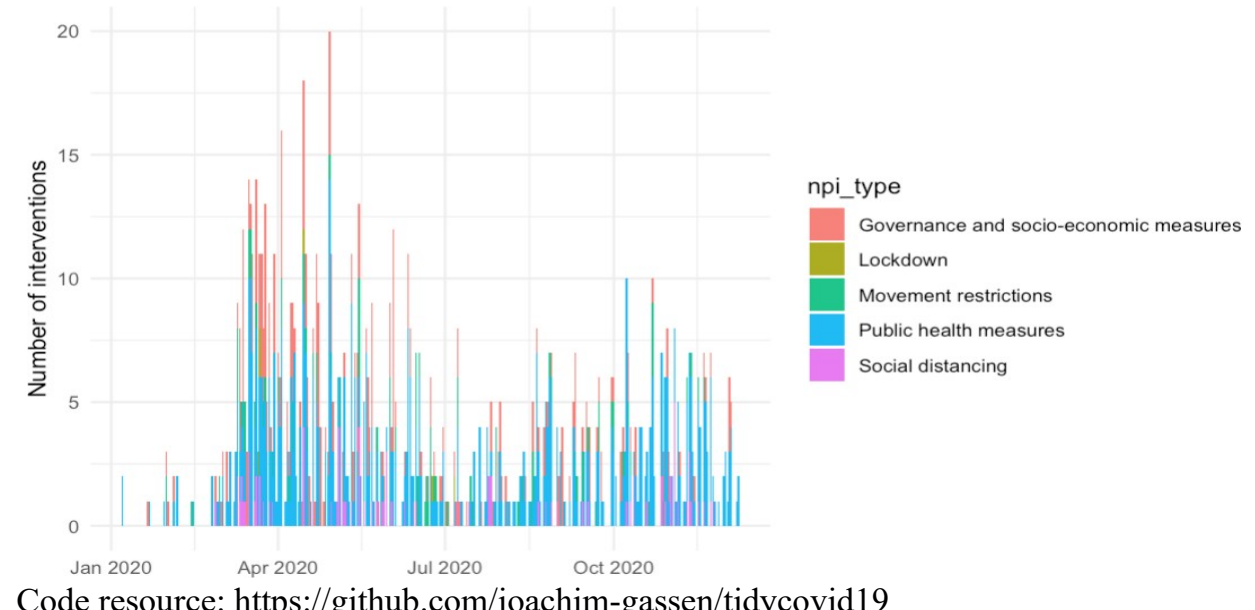

Figure 3 shows that both public health and governance and socio-economic measures were taken earlier as anticipatory measures (i.e., those implemented before the day when 10 cases were reported), and they have been used as early (i.e., those implemented the day of having between 10 and 200 cases) and late measures (i.e., those implemented after the day of having 200 cases). Throughout the observed period, public health measures have been the most preferred as the governments were inclined to taking less 
intrusive measures. Unlike public health measures, socio-economics measures were carefully used because these measures can trap countries' economies in self-imposed malfunction. Social distancing and movement restrictions measures were implemented as early measurements, but social distancing was taken slightly later than movement restrictions. Lockdown measures were reluctantly used as late measurements due to the ravage consequences on the economic factors but could not be a long-term solution. As the pandemic persisted in the long run, lockdown measures were considered only in South Korea and Germany (Tier 6).

\section{FIGURE 3 \\ THE PERCENTAGE SHARES OF INTERVENTION TYPES}

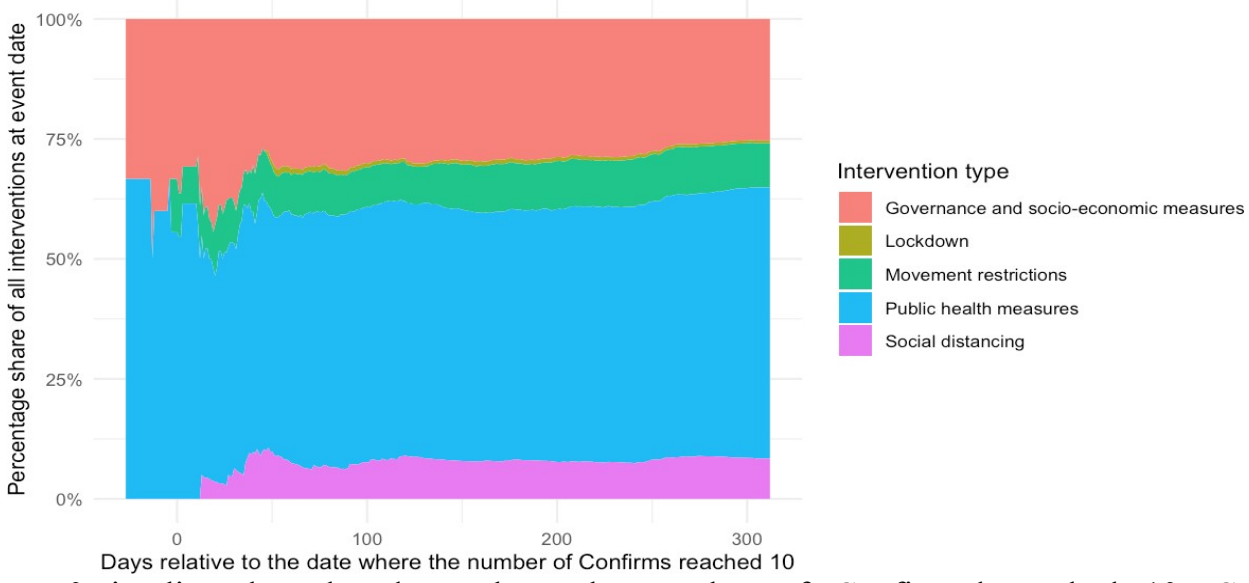

Note that $\mathrm{t}=0$ implies that the date where the number of Confirmed reached 10. Code resource: https://github.com/joachim-gassen/tidycovid19

As shown in Figure 4, Sweden took governance and socio-economic measures as anticipatory measures and used them as one of the primary government interventions over the observed period. Both public health measures and social distancing were taken as early measures, and movement restrictions were used as late measures. The proportion of public health measures was constantly 50\% starting from the 50 days after detecting the 10th cases. The proportion of governance and socio-economic measures decreased as the pandemic lasted in the long run. Instead, Sweden replaced governance and socio-economic measures with movement restrictions and social distancing. Interestingly, Sweden did not implement lockdown measures over the observed period.

South Korea implemented public health (40\%), movement restriction (40\%), social distancing (15\%), and lockdown (5\%) measures as early measures, but lockdown was implemented slightly later than the other two approaches. Starting from the 100 days when the massive virus test systems, including drive-thru testing, were initiated, the South Korean government minimized using lockdown measures. Instead, the country started to implement governance and socio-economic measures. The South Korean strategy can be simplified as the implementation of repeated cycles of test and contact trace measures. Unlike other countries, there has also been heavy use of surveillance technology: closed-circuit television (CCTV) and tracking of bank cards and mobile phone usage, to identify who to test in the first place. Despite the successful control for the battle against the pandemic, this trackdown approach has been obtaining caution due to the risk of threatening personal privacy, and so the other three countries did not implement this approach.

Following the South Korean success, Germany has been forced to pursue various government interventions to bend the confirmed and death curves. Since the country did not implement trackdown due to the privacy issue, the proportions of government interventions are more diverse than those in South Korea. Unlike South Korea in the same tier (Tier 6), Germany implemented governance and socioeconomic measures as primary early measures, starting from the 40 days. Lockdown measures were used 
from the 50 days but were not heavily enforced. As the pandemic persisted in the long run, the proportion of public health measures increased, whereas social distancing slightly decreased.

From Table 2, we can see that the United States significantly implemented late measures. Considering the 30 days to be the starting time of late measures, it is shown that the United States have heavily implemented public health and governance and socio-economic measures as both early and late measures. As an additional early and late measures, movement restrictions were used over the observed period. However, there is no evidence the country used lockdown measures that is a total closure of the economy. Even though social distancing is less than $5 \%$ out of the total government interventions, it is constantly detected as one of the late measures.

FIGURE 4

COMPARISON OF THE BREAK-DOWN OF INTERVENTION TYPES

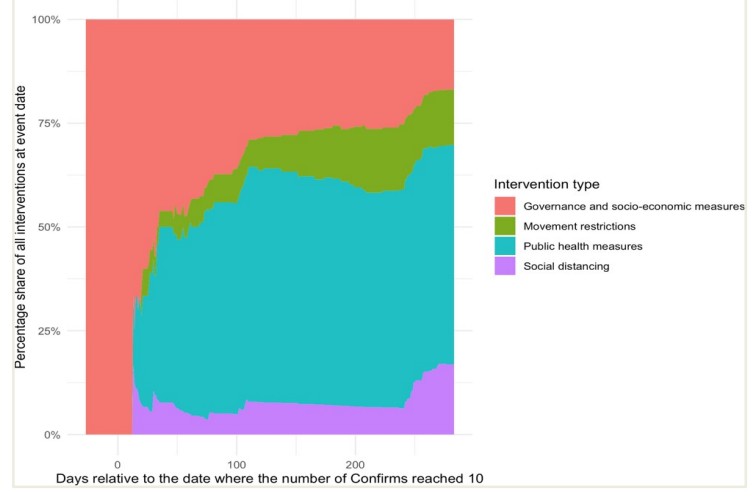

(a) Sweden

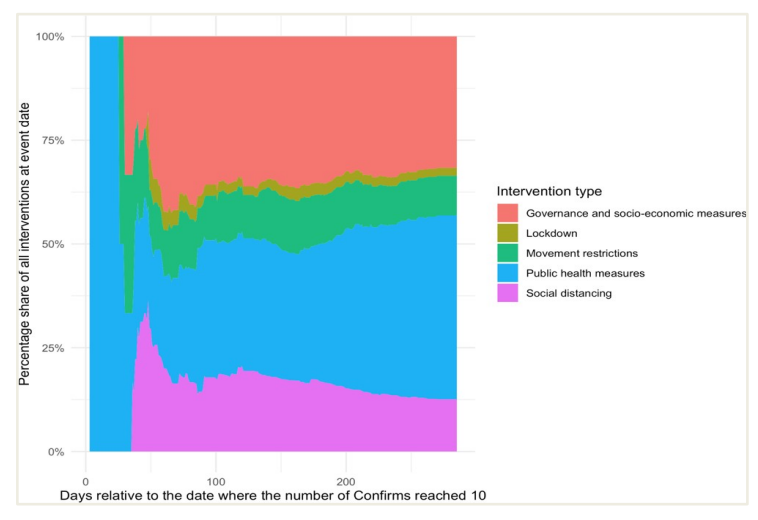

(c) Germany

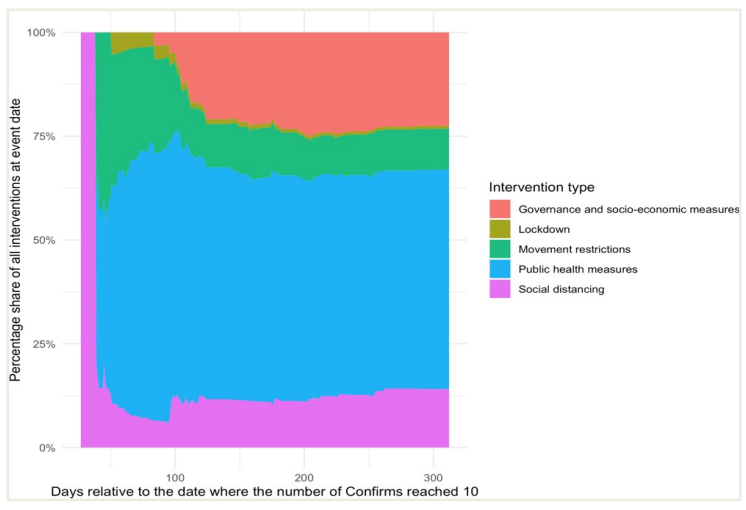

(b) South Korea

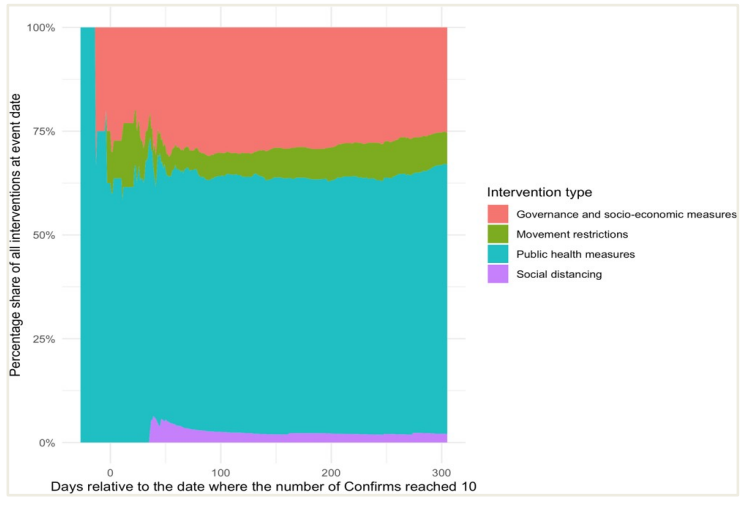

(d) United States

\section{SIR Estimation Results}

\section{Scenario Studies}

From the SIR model in Equation (3), we know that the rate of increase in the number of infections depends on the reproduction rate (i.e., R0), and especially the virus will die out as long as R0<1. It implies that the fewer susceptible people and the more people who are recovered and hence immune, the less our random interactions result in infections. Figure 5 shows that the ratios of confirmed cases and their cumulative cases over 16 months under different values of R0 $(=1.60,1.88,2.16,2.44,2.72,3.00)$. Considering that the health system can be overwhelmed when the fraction exceeds $1 \%$, it appears to take about 150-450 days to reach the threshold when R0 $>1.6$, with 250-450 days to reach the peak points. 
Figure 6-(b) depicts that $60 \%$ of the population are exposed to the virus within 450 days under almost all scenarios considered. Only under that case of $\mathrm{R} 0=1.6$, the virus is managed under control over the 500 days period, and so the government interventions can be effective to save the time that a vaccine becomes available. It implies that the objective of the government interventions is to maintain R 0 to be as close as possible to 1 .

\section{FIGURE 5}

\section{ESTIMATES OF CONFIRMED CASES AS A FRACTION OF POPULATION (N = 1000)}
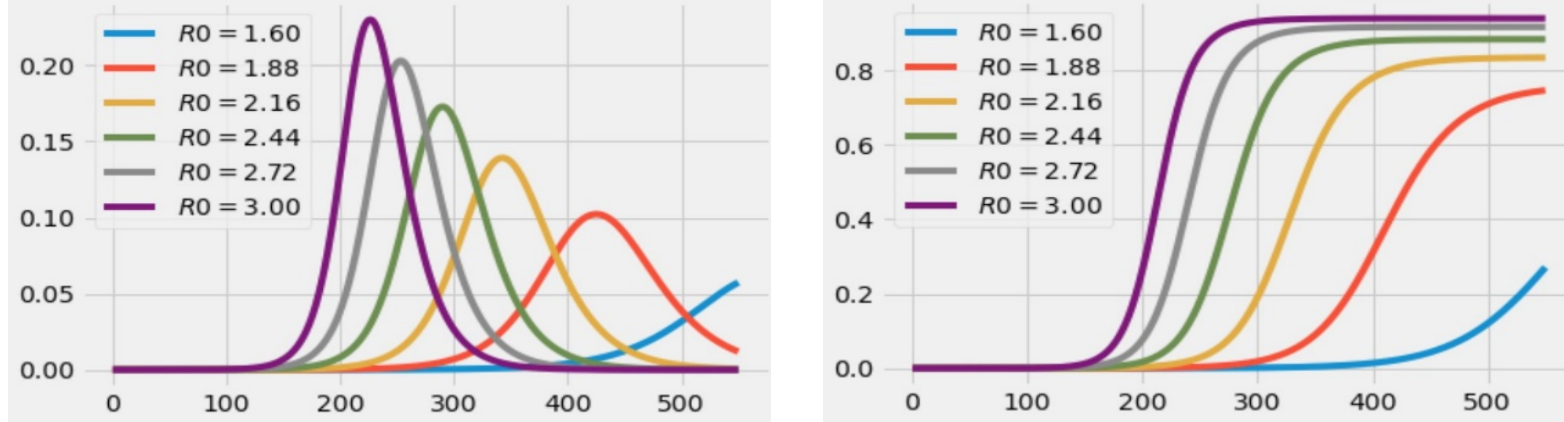

(a) The proportion of Estimated Confirmed Cases (b) The proportion of Estimated Cumulative Confirmed Cases

\section{Regression Results}

As shown in Figure 6, the cumulative development of confirmed cases and deaths (in a log scale) dramatically changed in the first 30-50 days and seemingly reached the saturated points in the 200 days. As a result, we estimated $\mathrm{R} 0$ for one month (i.e., R0(30)) the first case was observed, for 200 days (i.e., R0(200)) after the day when the first case was observed, and for the entire sample period (i.e., R0(t)), separately. The estimation was proceeded in the context of minimizing the difference between the observed and simulated confirmed cases.

\section{FIGURE 6 \\ ESTIMATES OF CONFIRMED CASES AND DEATHS}

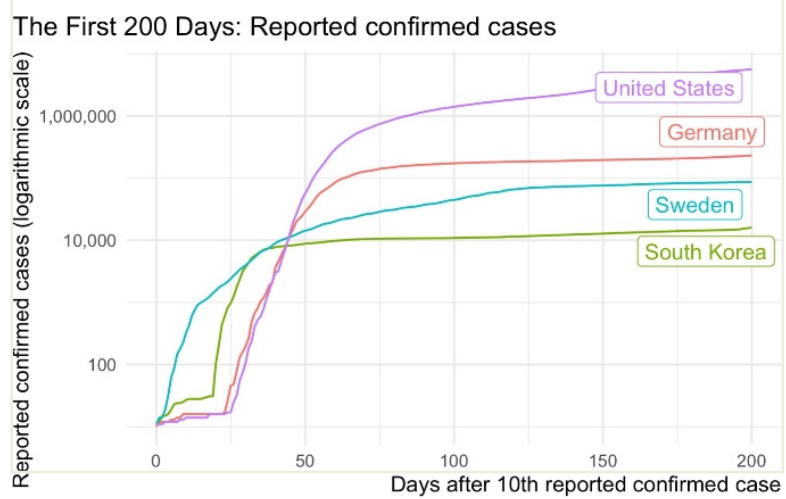

(a) Reported Confirmed Cases

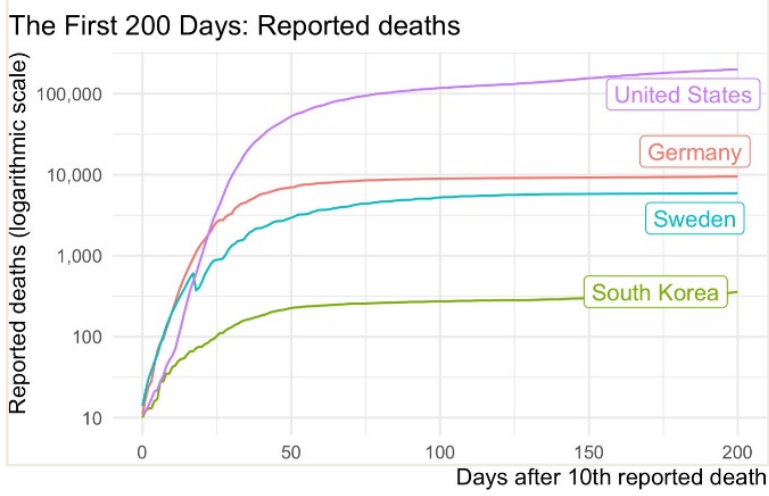

(b) Reported Deaths

The cumulative incidents from the day of the 10th case $(t=0)$ to the day after the 200th case was observed $(t=200)$. Data resource: JHU CSSE. Code resource: https://github.com/joachim-gassen/tidycovid19

Table 3 shows that in the first 30 days, various ranges of $R$ (values (ranged from 1.17 to 1.36) were detected across the countries where the highest R0 was found in South Korea, and the lowest value was found in Sweden. In the first 200 days, each government actively fought against the virus, and therefore we 
were able to see noticeable improvements in lowering the R0 values. Interestingly, in the 200 days, the lowest R0 was found in South Korea, and it implies that movement restrictions and public health measures implemented in South Korea were very effective between 30 days and 200 days. In the overall period, it appears that the South Korean government has done a great job in sustaining R0 to be close to 1 . This result is also confirmed in Figure 6.

Our results suggested the intense government interventions have successfully controlled the outbreak and transmission of COVID-19 between the 30 days and the 200 days period. However, the more interesting findings are observed in Sweden, where the herd immunity approach was implemented. Compared to other countries that implemented the vigorous interventions such as lockdown and movement restrictions, there is no evidence that Sweden's herd immunity approach brought total failure to control the virus. The Swedish example gives us the idea for small population countries that taking soft government interventions such as social distancing and public health measures can also effectively reduce the spread of COVID-19.

TABLE 3

RO COMPARISON OVER THE PERIODS, $\mathrm{t}=30$, 200, AND OVERALL

\begin{tabular}{|c|c|c|c|c|c|c|}
\hline Country & $\begin{array}{c}\text { Intervention } \\
\text { type }\end{array}$ & Population & $R((30)$ & $R((200)$ & $R((t)$ & $\begin{array}{c}\text { Expected } \\
\text { Peak }\end{array}$ \\
\hline Sweden & Herd immunity & $10,278,887$ & 1.17 & 1.15 & 1.18 & $2020-08-26$ \\
\hline South Korea & Trackdown & $51,709,098$ & 1.36 & 1.10 & 1.08 & $2020-09-08$ \\
\hline Germany & Lockdown & $83,092,962$ & 1.28 & 1.12 & 1.19 & $2020-09-20$ \\
\hline United States & Lockdown & $328,239,523$ & 1.25 & 1.20 & 1.31 & $2020-10-05$ \\
\hline
\end{tabular}

Figure 7 presents the simulated trajectories of the evolution of COVID-19 under the assumption that $R((=R((200))$ is constant across the 500 days. These include estimated cumulative susceptible (S; black), cumulative confirmed cases (I; red), and removed or recovered cases (R; green) in 500 days periods, along with the observed cases (blue) in 298 days sample periods. It appears to take about 208-248 days to reach the peak points. Regardless of the relative R0 similarities between the countries, almost one month difference of the peak dates between Sweden and Germany is expected due to the population size difference. Due to a large number of populations, the United States is expected to have the stagged peak point within the first wave of COVID-19. The model predictions for the peak date could be fitted to the observed peaked data reasonably well for Sweden and the Unites States. But for South Korea and Germany, the actual number of infections fell more rapidly than the model prediction, which is an indication of the success of the measures implemented by the governments. It implies that effective pandemic containment is possible, even in the absence of herd immunity in communities.

\section{FIGURE 7}

\section{SIMULATED TRAJECTORIES VS. OBSERVED INCIDENTS OF THE SPREAD OF COVID-19}

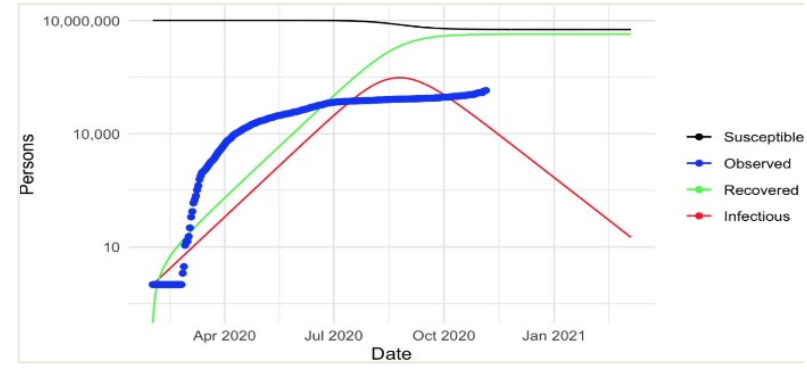

(a) Sweden

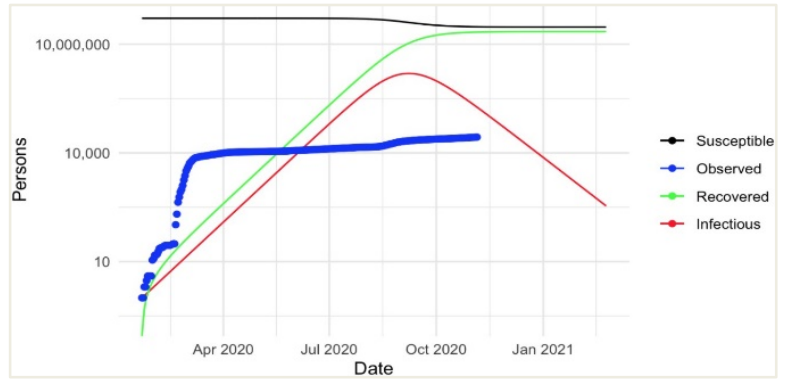

(b) South Korea 


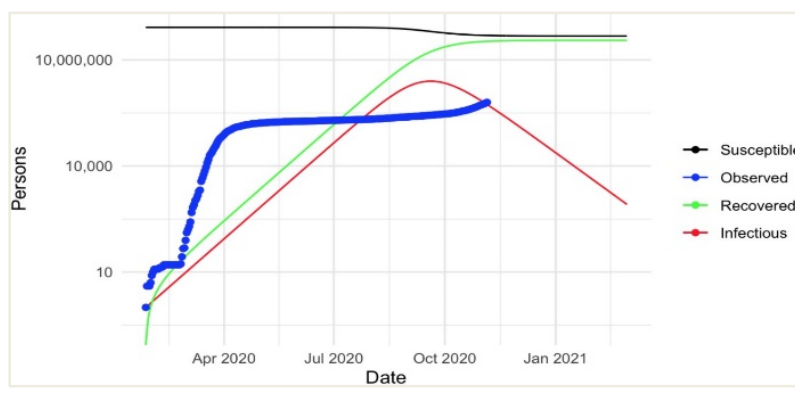

(c) Germany

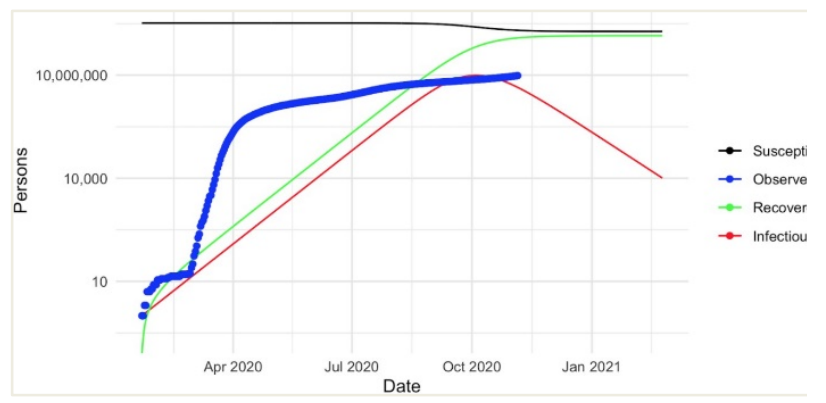

(d) United States

\section{CONCLUSION}

In this work, we investigated the effectiveness of several government interventions implemented in Sweden, South Korea, Germany, and the Unites States in the context of SIR model to provide insights into the spread of COVID-19 in communities. We began by presenting the basic SIR model and its mathematical analysis. The major problem with all infectious disease models is that the contact pattern in the population is often unknown, and somewhat difficult to measure ([26] Krämer, A. et al., 2009; [27] Giesecke, 2017). The SIR model used here is only a simple one and thus, the predictions that come out might not be accurate enough, something that also depends on the observed data and their trustworthiness. However, as the model data show, one thing that is certain is that COVID-19 is not going to go way quickly or easily.

It is inevitably true that the SIR model provides the fundamentals of getting a better understanding of the transmission of COVID-19. Moreover, the current infectious disease models are all extensions of the SIR model, estimating the parameters in the model is meaningful to obtain the better understanding of the dynamics of infection spread in a population. Then, we added the optimization procedures to estimate the parameters of the SIR system of equations and simulated the spread of COVID19 in the first wave (from January 22nd to November 5th, 2020) period. By comparing not only the different R0 values over the period but also the observed data with predictions, we examined the success of the three government intervention types: herd immunity, trackdown, and lockdown.

The effectiveness of the three approaches have been mixed. Our results confirmed the adequate measures have to be implemented to further prevent and control the spread of COVID-19. The South Korean government took an effective step to act in controlling the spread of the virus between 30 days and 200 days. The noticeable measures used in South Korea are isolation of susceptible individuals to avoid mixing them with no-symptoms and self-quarantine individuals, repeated cycles of test, and contact trace measures. It is also shown that the strong lockdown measures should be used within the first 90 days from the day when the 10th confirmed cases were found. The governance and socio-economic measures can be vigilant risk measures to harm the economy and so should be carefully implemented. Both social distancing and public health measures such as hand sanitization and mask mandating were mostly implemented as early as possible to control the high spread of COVID-19.

The long term 'exit strategy' needs to consider and balance not only the health outcomes, but also the economic consequences of any course of action. Given the caveats about possible variants of COVID-19, every effort should be made to distribute a safe and effective vaccine in the shortest possible time frame. Although competition drives invention and efficiency of a vaccine, we are facing a global problem that excessive rivalry may hamper a coordinated global effort that will provide community protection. 


\section{REFERENCES}

Anderson, R.M., \& May, R.M. (1982). Directly transmitted infectious diseases: Control by vaccination. Science, 215(4536), 1053-1060. New York, N.Y. https://doi.org/10.1126/science.7063839

Anderson, R.M., Heesterbeek, H., Klinkenberg, D., \& Hollingsworth T.D. (2020). How will countrybased mitigation measures influence the course of the COVID-19 epidemic? Lancet, 395, 931934.

Barro, R.J., Ursúa, J.F., \& Weng, J. (2020). The Coronavirus and the Great Influenza Pandemic: Lessons from the "Spanish Flu" for the Coronavirus's Potential Effects on Mortality and Economic Activity. NBER Working Papers, 26866. National Bureau of Economic Research, Inc.

Chan, J.F.W., Yuan, S., Kok, K.H., To, K.K.W., Chu, H., Yang, J., . . Yuen, K.Y. (2020). A familial cluster of pneumonia associated with the 2019 novel coronavirus indicating person-to-person transmission: A study of a family cluster. Lancet, 395(10223), 514-523. London, England. https://doi.org/10.1016/S0140-6736(20)30154-9

Cheng, C., Barceló, J., Hartnett, A.S., Kubinec, R., \& Messerschmidt, L. (2020). COVID-19 Government Response Event Dataset (CoronaNet v1.0). Nature Human Behavior, 4(7), 756-768.

Desvars-Larrive, A., Dervic, E., Haug, N., Niederkrotenthaler, T., Chen, J., di Natale, A., . . Thurner, S. (2020). A structured open dataset of government interventions in response to COVID-19. Scientific Data, 7, 285. https://doi.org/10.1038/s41597-020-00609-9

Fanelli, D., \& Piazza, F. (2020). Analysis and forecast of COVID-19 spreading in China, Italy and France. Chaos, Solitons, And Fractals, 134, 109761. https://doi.org/10.1016/j.chaos.2020.109761

Giesecke, J. (2017). Modern Infectious Disease Epidemiology (3rd edition). CRC Press.

Hale, T., Angrist, N., Goldszmidt, R., Kira, B., Petherick, A., Phillips, T., . . Tatlow, H. (2020). Oxford COVID-19 Government Response Tracker. Blavatnik School of Government. Retrieved from https://www.bsg.ox.ac.uk/research/research-projects/coronavirus-government-response- tracker

Hui, D. S., i Azhar, E., Madani, T.A., Ntoumi, F., Kock, R., Dar, O., . . Petersen, E. (2020). The continuing 2019-nCoV epidemic threat of novel coronaviruses to global health - The latest 2019 novel coronavirus outbreak in Wuhan, China. International Journal of Infectious Diseases, 91, 264-266.

Krämer, A., Akmatov, M., \& Kretzschmar, M. (2009). Principles of Infectious Disease Epidemiology. Modern Infectious Disease Epidemiology: Concepts, Methods, Mathematical Models, and Public Health, pp. 85-99. https://doi.org/10.1007/978-0-387-93835-6_5

Kucharski, A.J., Russell, T.W., Diamond, C., Liu, T., Edmunds, J., Funk, S., . . on behalf of the Centre for Mathematical Modelling of Infectious Diseases COVID-19 working group. (2020). Early dynamics of transmission and control of COVID-19: A mathematical modelling study. The Lancet Infectious Diseases, 20(5), 553-558. https://doi.org/10.1016/S1473-3099(20)30144-4

McKibbin, W.J., \& Fernando, R. (2020). Global Macroeconomic Scenarios of the COVID-19 Pandemic. CAMA Working Paper No. 62/2020. http://dx.doi.org/10.2139/ssrn.3635103

Pan, A., Liu, L., Wang, C., Guo, H., Hao, X., Wang, Q., . . Wu, T. (2020). Association of public health interventions with the epidemiology of the COVID-19 outbreak in Wuhan, China. JAMA, 323, 1915-1923.

Public Health Agency of Sweden. (2020). Initial Results from Ongoing investigation of antibodies to covid-19 virus (May report). Retrieved from https://www.folkhalsomyndigheten.se/the-publichealth-agency-of-sweden/

Rachah, A., \& Torres, D.F.M. (2015). Mathematical Modelling, Simulation, and Optimal Control of the 2014 Ebola Outbreak in West Africa. Discrete Dynamics in Nature and Society, p.842792. https://doi.org/10.1155/2015/842792

Sjödin, H., Johansson, A.F., Brännström, Å., Farooq, Z., Kriit, H.K., Wilder-Smith, A., . . Rocklov, J. (2020). COVID-19 healthcare demand and mortality in Sweden in response to nonpharmaceutical mitigation and suppression scenarios. International Journal of Epidemiology, 49(5), 1443-1453. https://doi.org/10.1093/ije/dyaa121 
Ugarov, A. (2020). Inclusive costs of NPI measures for COVID-19 pandemic: Three approaches. medRxiv. Preprint at https://doi.org/10.1101/2020.03.26.20044552

Venkatasen, M., Mathivanan, S.K., Jayagopal, P., Mani, P., Rajendran, S., Subramaniam, U., . . . Sorakaya Somanathan, M. (2020). Forecasting of the SARS- CoV-2 epidemic in India using SIR model, flatten curve and herd immunity. Journal of Ambient Intelligence and Humanized Computing, pp. 1-9. Advance online publication. https://doi.org/10.1007/s12652-020-02641-4

Wang, H., Wang, Z., Dong, Y., Chang, R., Xu, C., Yu, X., . . Cai, Y. (2020). Phase-adjusted estimation of the number of Coronavirus Disease 2019 cases in Wuhan, China. Cell Discovery, 6(1). https://doi.org/10.1038/s41421-020-0148-0

Wangping, J., Ke, H., Yang, S., Wenzhe, C., Shengshu, W., Shanshan, Y., . . Yao, H. (2020). Extended SIR Prediction of the Epidemics Trend of COVID-19 in Italy and Compared With Hunan, China. Frontiers in Medicine, 7, 169. https://doi.org/10.3389/fmed.2020.00169

World Health Organization. (2020). Novel Coronavirus (2019-nCoV) Situation Report 22. Retrieved from https://apps.who.int/iris/handle/10665/330991

World Health Organization. (2020). Pneumonia of unknown cause-China, WHO. Retrieved from https://www.who.int/csr/don/05-january-2020-pneumonia-of-unkown- cause-china/en/

Wu, J.T., Leung, K., \& Leung, G.M. (2020). Nowcasting and forecasting the potential domestic and international spread of the 2019-nCoV outbreak originating in Wuhan, China: A modeling study. Lancet, 395(10225), 689-697. London, England. https://doi.org/10.1016/S0140-6736(20)30260-9

Zhang, Z. (2007). The outbreak pattern of SARS cases in China as revealed by a mathematical model. Ecological Modelling, 204(3), 420-426. https://doi.org/10.1016/j.ecolmodel.2007.01.020

Zheng, Q., Jones, F.K., Leavitt, S.V., Ung, L., Labrique, A.B., Peters, D.H., . . Azman, A.S. (2020). HIT-COVID: A global database tracking public health interventions to COVID-19. Scientific Data, 7, 286. https://doi.org/10.1038/s41597-020-00610-2 